\title{
A public good model with lotteries in large groups*
}

\author{
Antonio Cabrales ${ }^{\dagger}$ \\ University College London and CEPR \\ Haydée Lugo ${ }^{\ddagger}$ \\ Universidad Complutense de Madrid
}

March 2015

\begin{abstract}
We analyze the effect of a large group on a public goods model with lotteries. We show that as populations get large, and with preferences in which individuals only care about their private consumptions and the total supply of the public good, the level of contributions converges to the one given by voluntary contributions. With preferences of the warm glow type, the contributions converge to a level strictly higher than those given by voluntary contributions, even though in general they do not yield first-best levels.
\end{abstract}

JEL-Classification: D64, H21, H41

Key-words: lotteries, public good, warm glow, efficiency.

\footnotetext{
${ }^{*}$ Cabrales acknowledges the support the Spanish Ministry of Science and Technology under grant ECO2012-34581. Lugo acknowledges financial support from the Spanish Ministry of Science and Innovation under grant ECO2013-42710-P.

${ }^{\dagger}$ Corresponding author. Address: 30 Gordon St., WC1H 0AX London, UK. Phone: +44 2031085229. Email: a.cabrales@ucl.ac.uk

¥Address: Department of Quantitative Economics (FAE II). UCM. Campus of Somosaguas, 28223 Madrid, Spain. Phone: +34 3942360. Email: hlugo@ccee.ucm.es
} 


\section{Introduction}

Most public goods in modern economies are provided by the government and funded from revenues obtained via general taxes. But coercive taxation has its limits, for reasons that have to do both with the inefficient (or second-best) way in which it is collected, and for political economy reasons. Yet, some legitimate needs are not covered by the ordinary revenues from the state, and both private and public entities resort to other mechanisms to fund those public goods. As it is well known (e.g. Bergstrom, Blume, and Varian (1986)), providing them via voluntary contributions usually leads to inefficient outcomes, so it is not surprising that human ingenuity has devised other means to achieve the goal of providing public goods efficiently.

One such method is a lottery in which a prize is given to the winner(s), but a fraction of the proceeds goes to the provision of public goods. For a while, there was a theoretical controversy about the usefulness of lotteries to improve efficiency (see e.g. Borg, Mason, and Shapiro (1991)) or equity (see e.g. Clotfelter and Cook (1989)) in public goods provision. Recently, Giebe and Schweinzer (2014) have shown that a "tax lottery" can correct the distortion on private consumption while, at same time, inducing efficient provision of public good and balancing the government's budget. The controversy was essentially settled when Morgan (2000) showed that lotteries can be used effectively to solve the problem. He proved that lotteries significantly increase the level of contributions above the one given by voluntary contributions. He also showed that for large enough prizes, the lottery could make the provision of the public good close to the first-best levels. An extension that relaxes the quasi-linearity assumption of Morgan was analyzed by Duncan (2002). He showed that with general utility functions, unlike in the quasi-linear case, a raffle has two opposite effects on the supply of a public good. First, it lowers the marginal price of contributing, which leads to an increase in the supply of public good. And second, it introduces uncertainty about the marginal utility of private consumption, which may lead to a decrease in the supply of the public good. Hence, with general utility functions, a raffle can increase or decrease the equilibrium supply of a public good, depending on the value of the prize. In both Morgan (2000) and Duncan (2002) the effect of the size of the prize on the provision of the public good is analyzed for a finite size of the population. 
Several papers confirm in the laboratory and in the field the theoretical prediction that lotteries outperform the voluntary contributions in terms of public good provision, Morgan and Sefton (2000), Orzen (2008), Schram and Onderstal (2009), Corazzini, Faravelli, and Stanca (2010), Duffy and Matros (2012) among others. Some of these papers have also revealed an excessive voluntary contribution relative to the equilibrium prediction with selfish agents. This finding indicates that people have preferences different from the selfish ones.

The aim of this paper is to establish the limits to the usefulness of lotteries in the provision of public goods even in the case in which the prize and the population can vary in the same proportion. We show first that as populations get large and with standard preferences, i.e. when an individual's utility depends on her private consumption and the total supply of the public good, ${ }^{1}$ the provision of public good converges to the (inefficient) one given by voluntary contributions. $^{2}$ A more positive result arises when one considers a model in which individuals are concerned not only about their private consumptions and total supply of the public good, but also about their own contributions. ${ }^{3}$ In this case, the contribution to the public good enters the utility function twice, once as a part of the public good and as a private good. ${ }^{4}$ As Bergstrom, Blume, and Varian (1986) stated individuals with such preferences feel a warm glow from having "done their bit", so we call them, warm-glow preferences. In large populations, when people have these preferences the lottery leads the contributions to converge to a level strictly higher than those given by voluntary contributions (still under warm glow preferences), even though in general they do not yield first-best levels.

Our results clarify why it is so important that lottery proceeds are earmarked to worthy causes, where warm glow is likely to be larger. ${ }^{5}$ In this way we shed light on a controversy about the meaningfulness of earmarking (see e.g. Buchanan (1963) and Borg and Mason (1988)) because of the fungible nature of

\footnotetext{
${ }^{1}$ These preferences are called by Andreoni (1990) purely altruistic.

${ }^{2}$ Olszewski and Siegel (2013) provide a general way to find equilibrium outcomes of contests with a large (but finite) number of competitors and prizes. This is done by approximating the equilibrium with a particular incentive-compatible and individually rational mechanisms.

${ }^{3}$ These are called by Andreoni (1990) impurely altruistic preferences.

${ }^{4}$ Similar models of mixture public-private good were first introduced by Cornes and Sandler (1984, 1986) and Steinberg (1987).

${ }^{5}$ In the U.S., for example, Lotto revenues are earmarked for the provision of education (see Landry and Price (2007)).
} 
government revenues. They also explain why in general governments do not rely on lotteries for a large part of the revenue creation for public good provision.

Section 2 describes the reference benchmark model from Morgan (2000) and also introduces warm glow preferences into such model. Section 3 presents the results for large populations. Section 4 briefly concludes.

\section{The Reference Model}

We first recapitulate the results of Morgan (2000). He shows that his results for the provision of public goods by means of lotteries also apply in the more general case analyzed by Bergstrom and Cornes (1983). They provide a specification of preferences in which income effects are present and the public goods allocation decision is separate from distributional decisions. They argue that this is essentially equivalent to assuming that individual preferences can be represented as a quasi-concave utility function of the form ${ }^{6}$,

$$
U_{i}^{V C}=\left(\omega_{i}-x_{i}\right) H(G)+h_{i}(G),
$$

where $H(\cdot)>0, h_{i}^{\prime}(\cdot)>0, h_{i}^{\prime \prime}(\cdot) \leq 0,{ }^{7}$ and where $\omega_{i} \in \mathbb{R}_{+}$denotes the wealth of individual $i, x_{i}$ her contribution to the public good and $G \in \mathbb{R}_{+}$denotes the level of public good provided. Hence, $\omega_{i}-x_{i}$ denotes individual's consumption of the private good. Individuals decide their allocation between their consumption of private good and their contribution to the public good by maximizing their expected utility, subject to the constraint that $x_{i}<\omega_{i}$. For the first-best benchmark, the optimal public good provision, which we denote by $G^{*}$ solves

$$
\max _{G \in \mathcal{R}_{+}}\left(\sum_{i=1}^{n} \omega_{i}-G\right) H(G)+\sum_{i=1}^{n} h_{i}(G) .
$$

Let $\widehat{x} \equiv \sum_{i=1}^{n} x_{i}$ be the aggregate contributions and since they pay for the public good, $G=\widehat{x}$. The provision of public good by voluntary contributions,

\footnotetext{
${ }^{6}$ Bergstrom and Cornes (1983) also provide a recipe for constructing quasi-concave functions of this form and a diagnostic test to determine whether a given function of this form is quasi-concave.

${ }^{7}$ It seems natural that private good is complementary to the public good. Also, since in a large society the public good is likely to become large, a separable $\left(\omega_{i}-x_{i}\right)+H(G)$ term would lead to the utility of private good consumption becoming very small as compared to $H(G)$.
} 
denoted by $G^{V}$ is the equilibrium of the game in which each agent maximizes $U_{i}^{V C}$ with respect to her contribution $x_{i}$ noting that $G=\widehat{x}$.

In the lottery model of Morgan (2000), the utility function of agent $i$ takes into account that $x_{i} / \widehat{x}$ represents the probability that individual $i$ wins the prize. Since the sum of all wagers must pay for the prize $R$, the public good provision, denoted by $G^{L}$, is determined by the excess of wagers over the prize ${ }^{8}$, that is:

$$
G^{L}=\widehat{x}-R
$$

In this case the expected utility of agent $i$ is:

$$
U_{i}^{L}=\left(\omega_{i}-x_{i}+R \frac{x_{i}}{\widehat{x}}\right) H(\widehat{x}-R)+h_{i}(\widehat{x}-R) .
$$

The amount provided of the public good using the lottery scheme, $G^{L}$, is the equilibrium of the game in which each agent maximizes $U_{i}^{L}$. For simplicity of exposition we will assume that for all games $\Gamma$ we will describe in what follows

Assumption 1. The utility function $U_{i}^{\Gamma}$ that represents the preferences of individual $i$ :

1. is twice continuously differentiable and concave in the decision variable $x_{i}$.

2. it satisfies $\left.\frac{\partial U_{i}^{\Gamma}}{\partial x_{i}}\right|_{x_{i}=0, x_{j}=\omega_{j}}>0,\left.\frac{\partial U_{i}^{\Gamma}}{\partial x_{i}}\right|_{x_{i}=\omega_{i}}<0$.

Using 1 . in the assumption we can characterize equilibria using first order conditions, and using 2. we guarantee that such solutions are interior. We can now show as Morgan (2000) proved that

Proposition 1. When preferences between public and private goods satisfy Assumption 1:

1. a voluntary contributions mechanism underprovides public goods with respect to the first-best level, i.e. $G^{V}<G^{*}$.

\footnotetext{
${ }^{8}$ As in Morgan (2000) and Duffy and Matros (2012), in our setting, the public good is provided only when the wagers are sufficient to cover the cost of the prize, otherwise the lottery is called off and the wagers are refunded.

It is important that the prize $R$ is an amount fixed ex-ante. If the prize were a fraction of lottery revenues, the utility of the players would be isomorphic to the one with voluntary contributions and the lottery would not change the outcome of the game.
} 
2. the public goods provision under a lottery mechanism is higher than the public good provision under a voluntary contributions mechanism, i.e. $G^{V}<G^{L}$.

3. the public goods provision under lotteries converges to its first-best level as the size of the lottery prize grows to infinity, i.e. $G^{L} \rightarrow G^{*}$ as $R \rightarrow \infty$.

Proof. See the Appendix.

The reason why the lottery increases contributions is that in lottery generates a negative externality. The more one bets, the lower are the chances of others to win the jackpot. This negative externality exactly balances the positive externality of giving to the public good in the limit as $R \rightarrow \infty$.

\subsection{Incorporating the warm glow of giving in the reference model}

We now incorporate the warm glow approach into the reference model by assuming that individuals are concerned about their private consumptions, the total supply of the public good and their own contributions. Hence, individual preferences can be represented as follows,

\section{Assumption 2.}

$$
U_{i}^{w g}=\left(\omega_{i}-x_{i}\right) H(G)+h_{i}(G) g\left(x_{i}\right),
$$

where the function $g(\cdot)$ represents the warm glow of giving. ${ }^{9}$ Setting

$$
g\left(x_{i}\right)=g_{1} f\left(x_{i}\right)+g_{0},
$$

where $f^{\prime}(\cdot)>0$ and $f^{\prime \prime}(\cdot)<0$, imply that individuals experience diminishing marginal utility from the warm glow, the particular case where $g_{1}=0$ and $g_{0}=1$ corresponds to (1), the model used by Morgan (2000) to show the robustness of his results. For simplicity, from now on, we set $g_{0}=1$.

The provision of a public good by voluntary contributions when preferences are as in (2), denoted by $G^{w g}$, is the equilibrium of the game in which each agent maximizes $U_{i}^{w g}$. For this game we can show that:

\footnotetext{
${ }^{9}$ Similarly as what happens between private and public goods, it seems natural that warm glow is complementary to the public good. And as in the previous case, since in a large society the public good is likely to become large, a separable $h_{i}(G)+g\left(x_{i}\right)$ term would lead to warm glow utility $g\left(x_{i}\right)$ becoming very small as compared to $h_{i}(G)$.
} 
Proposition 2. Public goods provision by a voluntary contributions mechanism is higher under warm glow preferences (as described in Assumption 2) than under standard preferences (as described in (1)), i.e. $G^{V}<G^{w g}$.

Proof. See the appendix.

It is not hard to see why the introduction of warm glow increases the level of voluntary contributions. Individuals now partly internalize the externality that their contributions cause on other agents in the economy. Morgan and Sefton (2000) do a series of experiments, in which they use the voluntary contributions mechanism for public good provision, as well as the lottery mechanism of Morgan (2000). In their experiment they observe that the level of contributions are quite significantly higher than one would expect given individuals with standard preferences. As an explanation, the authors claim "for example, Andreoni's warm glow model can similarly explain excessive giving by allowing utility to depend on the act of giving itself."

In Temimi (2001) the author shows that the introduction of warm-glow affects both the equilibrium level as well as the efficient level of public good provision. The condition determining the efficient level of provision for the public good case requires as usual that the sum of the marginal rates of substitution (between the public good and the net private good) is equal to one. In our case this is true when,

$$
\sum_{i=1}^{n} \frac{\frac{\partial U_{i}^{w g}}{\partial G}}{\frac{\partial U_{i}^{w g}}{\partial\left(\omega_{i}-x_{i}\right)}-\frac{\partial U_{i}^{w g}}{\partial x_{i}}}=1 .
$$

Applied to the model in (2), the efficient level of public good provision under warm glow, $G^{w g *}$, is given by the solution to:

$$
\sum_{i=1}^{n} \frac{\left(\omega_{i}-x_{i}\right) H^{\prime}\left(G^{w g *}\right)+g\left(x_{i}\right) h_{i}^{\prime}\left(G^{w g *}\right)}{H\left(G^{w g *}\right)-h_{i}\left(G^{w g *}\right) g_{1} f^{\prime}\left(x_{i}\right)}=1 .
$$

Notice also that as equation (3) shows, since warm glow shifts up the private benefit from giving, the first best level of contributions is higher under warm glow. 


\subsection{Lottery in the warm-glow model and the efficient level of contributions}

Now we incorporate the lottery mechanism of Morgan (2000) into the above model of warm glow. Individual $i$ now chooses $x_{i}$ to maximize

$$
U_{i}^{w g L}=\left(\omega_{i}-x_{i}+R \frac{x_{i}}{\widehat{x}}\right) H(\widehat{x}-R)+h_{i}(\widehat{x}-R) g\left(x_{i}\right) .
$$

As before, wagers pay for the prize $R$, so the public good provision, denoted by $G^{w g L}$, is:

$$
G^{w g L}=\widehat{x}-R
$$

Proposition 3. For any given distribution of private good endowments, when preferences are of the warm glow type (as described in Assumption 2) the contributions to public goods are higher under a lottery mechanism than under a voluntary contributions mechanism, i.e. $G^{w g}<G^{w g L}$.

Proof. See the appendix.

The result of proposition 3 says that lotteries are helpful to partly internalize the externality of giving even when agents have warm glow preferences. This is also in agreement with the evidence in Morgan and Sefton (2000). The lottery mechanism increases the provision of public good over voluntary contributions not only in populations of individuals with standard preferences but also in populations with warm glow preferences.

\section{The case of large populations}

In this section, we identify the limits to the usefulness of lotteries to improve the provision of public goods. One result in Morgan (2000) shows that wagers in the unique equilibrium provide levels of public good close to first-best as the lottery prize increases. However, $R$ is only useful if chosen so that in an interior equilibrium the level of provision $\sum_{i=1}^{n} x_{i}-R$ is positive (where $x_{i}$ is the contribution for each person). That is, a lottery prize yielding social benefits in terms of the public good must have $R$ with $\sum_{i=1}^{n} x_{i}>R$.

Given the limitation of increasing the prize when the group size is finite and fixed, we analyze the effect of increasing the prize in proportion to the group size. We will show that the prize is an effective tool for the provision of the public good only in the case in which individuals reveal warm glow preferences. 


\subsection{The linear case}

We now illustrate the limitations of lotteries to improve the provision of public goods in the case in which the prize and the population can vary in the same proportion. To do this, we first show the relationship between the first-best level of public good provision and the one achieved with a lottery mechanism in the large population limit, when the size of the population and the prize grow at the same rate, the agents' utilities is as in Assumption 2, and $H(\cdot)$ and $h(\cdot)$ are increasing and linear functions.

Proposition 4. Suppose $\lim _{n \rightarrow \infty} R / n=\rho$ and $H(\cdot)$ and $h_{i}(\cdot)$ are increasing and linear functions for all $i$ and $f\left(x_{i}\right)$ is an isoelastic functions $f\left(x_{i}\right)=$ $\frac{1}{1-\alpha}\left(x_{i}\right)^{1-\alpha}$ (with $\left.\alpha=1 \Rightarrow f\left(x_{i}\right)=\ln x_{i}\right)$. Then for any equilibrium we have that

$$
\lim _{n \rightarrow \infty} \frac{1}{n}\left|G^{w g *}-G^{w g L}\right| \geq 0
$$

with equality holding only for $\alpha=1$.

Proof. See the appendix.

The proposition shows that with preferences of the warm glow type and with a prize growing in the same proportion as the group size, the public good provision is strictly higher under a lottery mechanism than under a voluntary contributions mechanism. It does not, however, yield the first-best level except for the case when the function $f(\cdot)=\ln (\cdot)$.

The intuition for this result comes from the fact that in a large population both $G^{w g *}$ and $G^{w g L}$ become very large. As a result of this, the terms in the first order condition that contain the public good are much larger than the rest, so that the first order condition for the efficient solution ignoring lower order terms is

$$
\lim _{n \rightarrow \infty} \frac{1}{n}\left(-H G^{w g *}+h_{i} G^{w g *} g_{1} f^{\prime}\left(x_{i}^{w g *}\right)\right)=0,
$$

and for the equilibrium with the lottery mechanism letting $\widehat{x}$ be the total contributions the first order condition ignoring lower order terms is

$$
\begin{aligned}
\lim _{n \rightarrow \infty} \frac{1}{n}\left(\left(\frac{R}{\widehat{x}}-1\right) H G^{w g L}+h_{i} G^{w g L} g_{1} f^{\prime}\left(x_{i}^{w g L}\right)\right) & = \\
\lim _{n \rightarrow \infty} \frac{1}{n}\left(-H G^{w g L}+h_{i} G^{w g L} g_{1} \frac{\widehat{x} f^{\prime}\left(x_{i}^{w g L}\right)}{\widehat{x}-R}\right) & =0 .
\end{aligned}
$$


Taking into account that in the limit the two public good levels coincide only if $\sum_{i=1}^{n}\left(x_{i}^{w g L}-\rho\right)=\sum_{i=1}^{n} x_{i}^{w g *}$, it is readily seen that the expressions (5) and (6) can only yield $\sum_{i=1}^{n}\left(x_{i}^{w g L}-\rho\right)=\sum_{i=1}^{n} x_{i}^{w g *}$ when $f^{\prime}(x)=1 / x$.

For simplicity we have assumed interior solutions $x_{i}>0$ for all $i$ in the economy. But solutions need not be interior in general. For example, when $f^{\prime}(\cdot)$ is a constant, one can show that only the type with largest $h_{i}$ would make positive contributions in the large population equilibrium. ${ }^{10}$ Our results then would hold restricted to that high type only.

But there are also relatively simple sufficient conditions for all contributions to be interior. Denote by $G$ the total amount of public good and by $G_{-i}$ the sum of contributions from individuals other than $i$, let also

$\Psi_{i}\left(G_{-i} ; R\right)=\left(\frac{R}{G_{-i}+R}-1\right) H\left(G_{-i}\right)+\omega_{i} H^{\prime}\left(G_{-i}\right)+h_{i}^{\prime}\left(G_{-i}\right) f(0)+h_{i}(G) g_{1} f^{\prime}(0)$.

The function $\Psi_{i}\left(G_{-i} ; R\right)$ represents the marginal utility to individual $i$ at $x_{i}=0$. It is clear that $\Psi_{i}\left(G_{-i} ; R\right)>0$ for all $i$ guarantees interiority for all $i$. This is trivially satisfied when, as in Proposition $4, \lim _{x_{i} \rightarrow 0} f^{\prime}\left(x_{i}\right)=\infty$.

\section{$3.2 \quad$ A more general model}

Now, let us consider a general case in which $H(\cdot)$ and $h_{i}(\cdot)$ are general increasing, differentiable and strictly concave functions. That is, $H^{\prime}(\cdot)>0, h_{i}^{\prime}(\cdot)>0$ and $H^{\prime \prime}(\cdot)<0, h_{i}^{\prime \prime}(\cdot)<0$. If we introduce lotteries in the proposed model, we obtain that:

$$
U_{i}^{w g L}=\left(\omega_{i}-x_{i}+R \frac{x_{i}}{\widehat{x}}\right) H(\widehat{x}-R)+h_{i}(\widehat{x}-R) g\left(x_{i}\right) .
$$

where, as before, the aggregate contributions is denoted by $\widehat{x}=\sum_{i=1}^{n} x_{i}$ and the prize increases at the same proportion to group size $\lim _{n \rightarrow \infty} R / n=\rho$. Then

Proposition 5. if $H(\cdot)$ and $h_{i}(\cdot)$ are such that $\lim _{y \rightarrow \infty} h_{i}(y) / H(y)=k_{i}$, we have that in equilibrium $\lim _{n \rightarrow \infty} \frac{\widehat{x}}{n}$ solves:

$$
\lim _{n \rightarrow \infty} \frac{\widehat{x}}{n}=\lim _{n \rightarrow \infty} \frac{1}{n} \sum_{i=1}^{n} f^{\prime-1}\left(\frac{\widehat{x}-R}{\widehat{x}} \frac{1}{g_{1} k_{i}}\right) .
$$

Proof. See the appendix.

\footnotetext{
${ }^{10} \mathrm{We}$ are grateful to a referee for this observation.
} 
As a result, if $g_{1}=0$ then $\widehat{x} / n$ approaches the corner solution $\widehat{x} / n=\rho$.

Again, the intuition for the result follows from the fact that in a large society the terms that matter for first order conditions are those where the public good is present. That is,

$$
\begin{aligned}
\lim _{n \rightarrow \infty} \frac{1}{n}\left(\left(\frac{R}{\widehat{x}}-1\right) H(\widehat{x}-R)+h_{i}(\widehat{x}-R) g_{1} f^{\prime}\left(x_{i}\right)\right) & = \\
\lim _{n \rightarrow \infty} \frac{1}{n}\left(\left(\frac{R}{\widehat{x}}-1\right)+\frac{h_{i}(\widehat{x}-R)}{H(\widehat{x}-R)} g_{1} f^{\prime}\left(x_{i}\right)\right) & =0 .
\end{aligned}
$$

The result follows immediately from this expression by using the fact that $\lim _{y \rightarrow \infty} h_{i}(y) / H(y)=k_{i}$.

In this section, we have shown that when the prize grows at the same rate as the group size in a large population, lotteries improve the provision of public goods if individuals have warm glow preferences, but not if they are selfish. But we also show that in general the first-best levels cannot be reached even with warm glow preferences.

Our results contribute to clarify why it is important to earmark lottery revenues, and how this should be done. The lotteries in which their proceeds are earmarked to finance worthy public goods are more likely to attract individuals with a taste for altruism, whose giving behavior yields them a warm glow. The linkage between earmarked lotteries and warm glow preferences seems then crucial to the success of lotteries as a means of financing public goods in large populations.

\section{Conclusions}

In this paper we have shown that lotteries have limits as a tool to achieve efficient public good provision in large populations. But we also show that lotteries are clearly more effective than voluntary contributions when individuals experience a warm glow of giving to public goods. One concrete empirical implication from our analysis is that goods likely to produce a warm glow are more likely financed in this way. This could be useful to analyze empirically the extent to which the effects characterized in this paper are present in the field. 


\section{References}

Andreoni, J. (1990): "Impure altruism and donations to public goods: A theory of warm-glow giving?," Economic Journal, 100(4), 464-477.

Bergstrom, T., L. Blume, and H. Varian (1986): "On the Private Provision of Public Goods," Journal of Public Economics, 29, 25-49.

Bergstrom, T., and R. Cornes (1983): "Independence of allocative efficiency from distribution in the theory of public goods," Econometrica, 51(6), 1753-1765.

Borg, M. O., And P. M. Mason (1988): "The Budgetary Incidence of a Lottery to Support Education," National Tax Journal, 61, 75-85.

Borg, M. O., P. M. Mason, and S. L. Shapiro (1991): The economic consequences of state lotteries. Praeger Press, New York, NY.

Buchanan, J. (1963): "The Economics of Earmarked Taxes," Journal of Political Economy, 71, 457-469.

Clotfelter, C. T., and P. P. Cook (1989): Selling hope, State lotteries in America. Harvard University Press, Cambridge, MA.

Corazzini, L., M. Faravelli, and L. Stanca (2010): "A prize to give for: An experiment on Public Good Funding Mechanism," Economic Journal, 120, 944-967.

Cornes, R., and T. Sandler (1984): "Easy riders, joint production and public goods," Economic Journal, 94, 580-98.

(1986): The Theory of Externalities, Public Goods and Club Goods. Cambridge U. P., 1st edn.

Duffy, J., And A. Matros (2012): "All-Pay Actions vs. Lotteries as Provisional Fixed-Prize Fundraising Mechanisms: Theory and Evidence," Discussion paper.

Duncan, B. (2002): "Pumpkin pies and public goods: The raffle fundraising strategy," Public Choice, 111, 49-71. 
Giebe, T., And P. Schweinzer (2014): "Consuming your way to Efficiency," European Journal of Political Economy, 36, 1-12.

Landry, C. E., and M. K. Price (2007): "Earmarking lottery proceeds for public goods: Empirical evidence from US lotto expenditures," Economics Letters, 95(3), 451-455.

Morgan, J. (2000): "Financing public goods by means of lotteries," The Review of Economic Studies, 67(4), 761-784.

Morgan, J., And M. Sefton (2000): "Funding public goods with lotteries: experimental evidence," The Review of Economic Studies, 67(4), 785-810.

Olszewski, W., and R. Siegel (2013): "Large Contests," Discussion paper, Mimeo.

Orzen, H. (2008): "Fundraising through competition: Evidence from the lab," CeDEx discussion paper series 2008-11, Nottingham.

Schram, A., and S. Onderstal (2009): "Bidding to Give: An Experimental Comparison of Actions for Charity," International Economic Review, 50(2), $431-457$.

SteinBerg, R. (1987): "Voluntary donations and public expenditures in a federalist system," American Economic Review, 77, 24-36.

Temimi, A. (2001): "Does altruism mitigate free-riding and welfare loss?," Economics Bulletin, 8(5), 1-8. 


\section{Appendix}

\section{Proof of Proposition 1}

Proof. This proposition is already shown in Morgan (2000), we merely add it here for completeness.

$G^{*}$ solves

$$
\sum_{i=1}^{n} h_{i}^{\prime}\left(G^{*}\right)=H\left(G^{*}\right)-H^{\prime}\left(G^{*}\right)\left(\sum_{i=1}^{n} \omega_{i}-G^{*}\right) .
$$

and we also obtain $G^{V}$ by adding first-order conditions of optimization problems for each agent $i$,

$$
\sum_{i=1}^{n} h_{i}^{\prime}\left(G^{V}\right)=n H\left(G^{V}\right)-H^{\prime}\left(G^{V}\right)\left(\sum_{i=1}^{n} \omega_{i}-G^{V}\right) .
$$

It is then easy to verify that $G^{V}<G^{*}$. Also $G^{L}$, solves the sum of first-order conditions.

$$
\sum_{i=1}^{n} h_{i}^{\prime}\left(G^{L}\right)=H\left(G^{L}\right)\left(n-(n-1) \frac{R}{R+G^{L}}\right)-H^{\prime}\left(G^{L}\right)\left(\sum_{i=1}^{n} \omega_{i}-G^{L}\right)
$$

Comparing expressions (8) and (9), Morgan (2000) remarks that the two expressions differ by the term associated with the negative externality of the lottery multiplied by $H(G)$. Thus, $G^{V}<G^{L}$. Taking the limit of (9) as $R \rightarrow \infty$, expression (9) becomes identical to (7).

\section{Proof of Proposition 2}

Proof. At an interior maximum, the first-order condition of (2) with respect to $x$,

$$
-H(G)+\left(\omega_{i}-x_{i}\right) H^{\prime}(G)+h_{i}^{\prime}(G)\left(g_{1} f\left(x_{i}\right)+1\right)+h_{i}(G) g_{1} f^{\prime}\left(x_{i}\right)=0
$$

The equilibrium level of public good provided by voluntary contributions with the presence of warm glow giving, solves the sum of first-order conditions,

$$
\begin{aligned}
\sum_{i=1}^{n} h_{i}^{\prime}\left(G^{w g}\right)\left(g_{1} f\left(x_{i}\right)+1\right)+\sum_{i=1}^{n} h_{i}\left(G^{w g}\right) g_{1} f^{\prime}\left(x_{i}\right) & =n H\left(G^{w g}\right) \\
& -H^{\prime}\left(G^{w g}\right)\left(\sum_{i=1}^{n} \omega_{i}-G^{w g}\right)
\end{aligned}
$$


Then, we have

$$
\begin{aligned}
\sum_{i=1}^{n} h_{i}^{\prime}\left(G^{w g}\right) & =n H\left(G^{w g}\right)-H^{\prime}\left(G^{w g}\right)\left(\sum_{i=1}^{n} \omega_{i}-G^{w g}\right) \\
& -\sum_{i=1}^{n} h_{i}^{\prime}\left(G^{w g}\right) g_{1} f\left(x_{i}\right)-\sum_{i=1}^{n} h_{i}\left(G^{w g}\right) g_{1} f^{\prime}\left(x_{i}\right)
\end{aligned}
$$

Compare expressions (8) and (11) to verify that the result holds. The two expressions differ by the term associated with the effect of the warm glow.

\section{Proof of Proposition 3}

Proof. Take the first-order conditions of (4) with respect to $x_{i}$ to find

$$
\begin{aligned}
\left(R \frac{\widehat{x}-x_{i}}{\widehat{x}^{2}}-1\right) H(\widehat{x}-R)+\left(\omega_{i}-x_{i}+R \frac{x_{i}}{\widehat{x}}\right) H^{\prime}(\widehat{x}-R) & + \\
h_{i}^{\prime}(\widehat{x}-R)\left(g_{1} f\left(x_{i}\right)+1\right)+h_{i}(\widehat{x}-R) g_{1} f^{\prime}\left(x_{i}\right) & =0
\end{aligned}
$$

The public goods provision $G^{w g L}$ solves the sum of the first-order conditions,

$$
\begin{aligned}
\sum_{i=1}^{n} h_{i}^{\prime}\left(G^{w g L}\right)\left(g_{1} f\left(x_{i}\right)+1\right)+\sum_{i=1}^{n} h_{i}\left(G^{w g L}\right) g_{1} f^{\prime}\left(x_{i}\right)= & H\left(G^{w g L}\right)\left(n-(n-1) \frac{R}{R+G^{w g L}}\right) \\
& -H^{\prime}\left(G^{w g L}\right)\left(\sum_{i=1}^{n} \omega_{i}-G^{w g L}\right)
\end{aligned}
$$

We have

$$
\begin{aligned}
\sum_{i=1}^{n} h_{i}^{\prime}\left(G^{w g L}\right)= & H\left(G^{w g L}\right)\left(n-(n-1) \frac{R}{R+G^{w g L}}\right)-H^{\prime}\left(G^{w g L}\right)\left(\sum_{i=1}^{n} \omega_{i}-G^{w g L}\right) \\
& -\sum_{i=1}^{n} h_{i}^{\prime}\left(G^{w g L}\right) g_{1} f\left(x_{i}\right)-\sum_{i=1}^{n} h_{i}\left(G^{w g L}\right) g_{1} f^{\prime}\left(x_{i}\right)
\end{aligned}
$$

Notice that expressions (11) and (12) differ by the term associated to the negative externality of the lottery multiplied by $H(G)$. Similarly as to the model without warm-glow, the public goods provision under the lottery is greater than under voluntary contributions. That is, $G^{w g}<G^{w g L}$.

\section{Proof of Proposition 4}


Proof. To obtain the optimal level of provision, we use the first order conditions from the problem

$$
\sum_{i=1}^{n} U_{i}^{w g}=\sum_{i=1}^{n}\left(\omega_{i}-x_{i}\right) H(G)+h_{i}(G)\left(g_{1} f\left(x_{i}\right)+g_{0}\right)
$$

which yield

$\left(\omega_{i}-x_{i}\right) H^{\prime}\left(G^{w g *}\right)+h_{i}^{\prime}\left(G^{w g *}\right)\left(g_{1} f\left(x_{i}\right)+g_{0}\right)-H\left(G^{w g *}\right)+h_{i}\left(G^{w g *}\right) g_{1} f^{\prime}\left(x_{i}\right)=0$

which using linearity of $H($.$) and h_{i}($.$) can be expressed as$

$$
\left(\left(\omega_{i}-x_{i}\right) H+h_{i}\left(g_{1} f\left(x_{i}\right)+g_{0}\right)-H G^{w g *}+h_{i} G^{w g *} g_{1} f^{\prime}\left(x_{i}\right)\right)=0
$$

We now take limits for $n$ large and assuming that $G^{w g *}$ is of $O(n)$ (something we later establish) we have

$$
\begin{gathered}
\lim _{n \rightarrow \infty}\left(\frac{1}{n}\left(\left(\omega_{i}-x_{i}\right) H+h_{i}\left(g_{1} f\left(x_{i}\right)+g_{0}\right)-H G^{w g *}+h_{i} G^{w g *} g_{1} f^{\prime}\left(x_{i}\right)\right)\right)=0 \\
\lim _{n \rightarrow \infty}\left(\frac{1}{n}\left(-H G^{w g *}+h_{i} G^{w g *} g_{1} f^{\prime}\left(x_{i}\right)\right)\right)=0
\end{gathered}
$$

which means that

$$
\lim _{n \rightarrow \infty}\left(f^{\prime}\left(x_{i}\right)\right)=\frac{H}{g_{1} h_{i}}
$$

so that

$$
\lim _{n \rightarrow \infty} \frac{1}{n} G^{w g *}=\lim _{n \rightarrow \infty} \frac{1}{n} \sum_{i=1}^{n} x_{i}=\lim _{n \rightarrow \infty} \frac{1}{n} \sum_{i=1}^{n} f^{\prime-1}\left(\frac{H}{g_{1} h_{i}}\right)
$$

when $f(x)=\ln (x)$

$$
\lim _{n \rightarrow \infty} \frac{1}{n} G^{w g *}=\lim _{n \rightarrow \infty} \frac{1}{n} \sum_{i=1}^{n} x_{i}=\lim _{n \rightarrow \infty} \frac{1}{n} \sum_{i=1}^{n}\left(\frac{g_{1} h_{i}}{H}\right)
$$

when $f(x)=\frac{1}{1-\alpha}(x)^{1-\alpha}$

$$
\lim _{n \rightarrow \infty} \frac{1}{n} G^{w g *}=\lim _{n \rightarrow \infty} \frac{1}{n} \sum_{i=1}^{n} x_{i}=\lim _{n \rightarrow \infty} \frac{1}{n} \sum_{i=1}^{n}\left(\frac{g_{1} h_{i}}{H}\right)^{\frac{1}{\alpha}}
$$

Note that this also establishes $G^{w g *}$ is of $O(n)$.

The first-order condition of (12) with respect to $x_{i}$ is,

$$
\left(R \frac{\widehat{x}-x_{i}}{\widehat{x}^{2}}-1\right) H(\widehat{x}-R)+\left(\omega_{i}-x_{i}+R \frac{x_{i}}{\widehat{x}}\right) H^{\prime}(\widehat{x}-R)+h_{i}^{\prime}(\widehat{x}-R)\left(g_{1} f\left(x_{i}\right)+1\right)+h_{i}(\widehat{x}-R) g_{1} f^{\prime}\left(x_{i}\right)=0
$$


using linearity this is equivalent to

$$
\left(R \frac{\widehat{x}-x_{i}}{\widehat{x}^{2}}-1\right) H(\widehat{x}-R)+\left(\omega_{i}-x_{i}+R \frac{x_{i}}{\widehat{x}}\right) H+h_{i}\left(g_{1} f\left(x_{i}\right)+1\right)+h_{i}(\widehat{x}-R) g_{1} f^{\prime}\left(x_{i}\right)=0
$$

We now take limits for $n$ large and assuming that $G^{w g L}$ is of $O(n)$ (something we later establish) we have

$$
\begin{gathered}
\lim _{n \rightarrow \infty}\left(\left(R \frac{\widehat{x}-x_{i}}{\widehat{x}^{2}}-1\right) H(\widehat{x}-R)+\left(\omega_{i}-x_{i}+R \frac{x_{i}}{\widehat{x}}\right) H+h_{i}\left(g_{1} f\left(x_{i}\right)+1\right)+h_{i}(\widehat{x}-R) g_{1} f^{\prime}\left(x_{i}\right)\right)=0 \\
\lim _{n \rightarrow \infty}\left(\left(\frac{R}{\widehat{x}}-1\right) H(\widehat{x}-R)+h_{i}(\widehat{x}-R) g_{1} f^{\prime}\left(x_{i}\right)\right)=0
\end{gathered}
$$

which means that

$$
\lim _{n \rightarrow \infty}\left(f^{\prime}\left(x_{i}\right)\right)=\frac{H}{g_{1} h_{i}}\left(1-\lim _{n \rightarrow \infty} \frac{R}{\widehat{x}}\right)
$$

when $f(x)=\ln (x)$

$$
\begin{aligned}
\lim _{n \rightarrow \infty} \frac{1}{x_{i}} & =\frac{H}{g_{1} h_{i}}\left(1-\lim _{n \rightarrow \infty} \frac{R}{\widehat{x}}\right) \\
\lim _{n \rightarrow \infty} x_{i} & =\frac{g_{1} h_{i}}{H}\left(\lim _{n \rightarrow \infty} \frac{\widehat{x}}{\widehat{x}-R}\right) \\
\lim _{n \rightarrow \infty} \frac{\widehat{x}}{n} & =\lim _{n \rightarrow \infty} \frac{\widehat{x}}{\widehat{x}-R} \frac{1}{n} \sum_{i=1}^{n} \frac{g_{1} h_{i}}{H} \\
\lim _{n \rightarrow \infty} \frac{\widehat{x}-R}{n} & =\lim _{n \rightarrow \infty} \frac{1}{n} \sum_{i=1}^{n} \frac{g_{1} h_{i}}{H}
\end{aligned}
$$


when $f(x)=\frac{1}{1-\alpha}(x)^{1-\alpha}$

$$
\begin{aligned}
\lim _{n \rightarrow \infty} x_{i}^{-\alpha} & =\frac{H}{g_{1} h_{i}}\left(1-\lim _{n \rightarrow \infty} \frac{R}{\widehat{x}}\right) \\
\lim _{n \rightarrow \infty} x_{i}^{\alpha} & =\frac{g_{1} h_{i}}{H}\left(\lim _{n \rightarrow \infty} \frac{\widehat{x}-R}{\widehat{x}-R}\right) \\
\lim _{n \rightarrow \infty} x_{i} & =\lim _{n \rightarrow \infty}\left(\frac{g_{1} h_{i}}{H}\right)^{\frac{1}{\alpha}}\left(\frac{\widehat{x}}{\widehat{x}-R}\right)^{\frac{1}{\alpha}} \\
\lim _{n \rightarrow \infty} \frac{\widehat{x}}{n} & =\lim _{n \rightarrow \infty}\left(\frac{\widehat{x}}{\widehat{x}-R}\right)^{\frac{1}{\alpha}} \frac{1}{n} \sum_{i=1}^{n}\left(\frac{g_{1} h_{i}}{H}\right)^{\frac{1}{\alpha}} \\
\lim _{n \rightarrow \infty}\left(\frac{\widehat{x}-R}{n}\right)^{\frac{1}{\alpha}} & =\lim _{n \rightarrow \infty}\left(\frac{\widehat{x}}{n}\right)^{\frac{1-\alpha}{\alpha}} \frac{1}{n} \sum_{i=1}^{n}\left(\frac{g_{1} h_{i}}{H}\right)^{\frac{1}{\alpha}} \\
\lim _{n \rightarrow \infty}\left(\frac{G^{w g L}}{n}\right)^{\frac{1}{\alpha}} & =\lim _{n \rightarrow \infty}\left(\frac{\widehat{x}}{n}\right)^{\frac{1-\alpha}{\alpha}} \frac{1}{n} \sum_{i=1}^{n}\left(\frac{g_{1} h_{i}}{H}\right)^{\frac{1}{\alpha}} \\
\lim _{n \rightarrow \infty}\left(\frac{G^{w g L}}{n}\right)^{\frac{1}{\alpha}} & =\lim _{n \rightarrow \infty}\left(\left(\frac{\widehat{x}}{n}\right)^{\frac{1-\alpha}{\alpha}} \frac{G^{w g *}}{n}\right)
\end{aligned}
$$

Assume, by way of a contradiction, that $G^{w g *}=G^{w g L}$,then

$$
\begin{aligned}
\lim _{n \rightarrow \infty}\left(\frac{G^{w g L}}{n}\right)^{\frac{1}{a}} & =\lim _{n \rightarrow \infty}\left(\left(\frac{\widehat{x}}{n}\right)^{\frac{1-\alpha}{\alpha}} \frac{G^{w g L}}{n}\right) \\
\lim _{n \rightarrow \infty}\left(\frac{G^{w g L}}{n}\right)^{\frac{1-\alpha}{a}} & =\lim _{n \rightarrow \infty}\left(\frac{\widehat{x}}{n}\right)^{\frac{1-\alpha}{\alpha}} \\
\lim _{n \rightarrow \infty}\left(\frac{G^{w g L}}{n}\right) & =\lim _{n \rightarrow \infty}\left(\frac{\widehat{x}-R}{n}\right)=\lim _{n \rightarrow \infty}\left(\frac{\widehat{x}}{n}\right)
\end{aligned}
$$

This entails a contradiction, and the result follows.

\section{Proof of Proposition 5}

Proof. The first-order condition,

$$
\begin{array}{r}
\left(R \frac{\widehat{x}-x_{i}}{\widehat{x}^{2}}-1\right) H(\widehat{x}-R)+\left(\omega_{i}-x_{i}+R \frac{x_{i}}{\widehat{x}}\right) H^{\prime}(\widehat{x}-R) \\
h_{i}^{\prime}(\widehat{x}-R)\left(g_{1} f\left(x_{i}\right)+1\right)+h_{i}(\widehat{x}-R) g_{1} f^{\prime}\left(x_{i}\right)=0
\end{array}
$$

where $\widehat{x}=\sum x_{i}$ noting that $H^{\prime}$ and $h^{\prime}$ are bounded by concavity and assuming 
that $\widehat{x}-R$ is of $O(n)$ (something we later establish)as $n \rightarrow \infty$, we obtain

$$
\begin{aligned}
& \lim _{n \rightarrow \infty}\left(\frac{1}{n}\left(\left(R \frac{\widehat{x}-x_{i}}{\widehat{x}^{2}}-1\right) H(\widehat{x}-R)+\left(\omega_{i}-x_{i}+R \frac{x_{i}}{\widehat{x}}\right) H^{\prime}(\widehat{x}-R)\right)+\right. \\
& \left.\frac{1}{n}\left(h_{i}^{\prime}(\widehat{x}-R)\left(g_{1} f\left(x_{i}\right)+1\right)+h_{i}(\widehat{x}-R) g_{1} f^{\prime}\left(x_{i}\right)\right)\right) \\
= & \lim _{n \rightarrow \infty} \frac{1}{n}\left(\frac{R}{\widehat{x}} H(\widehat{x}-R)-H(\widehat{x}-R)+h_{i}(\widehat{x}-R) g_{1} f^{\prime}\left(x_{i}\right)\right)=0 \\
= & \lim _{n \rightarrow \infty} \frac{1}{n}\left(\frac{R-\widehat{x}}{\widehat{x}} H(\widehat{x}-R)+h_{i}(\widehat{x}-R) g_{1} f^{\prime}\left(x_{i}\right)\right)=0
\end{aligned}
$$

so that

$$
\begin{aligned}
\lim _{n \rightarrow \infty} \frac{1}{n}\left(h_{i}(\widehat{x}-R) g_{1} f^{\prime}\left(x_{i}\right)\right) & =\lim _{n \rightarrow \infty} \frac{1}{n}\left(\frac{\widehat{x}-R}{\widehat{x}} H(\widehat{x}-R)\right) \\
\lim _{n \rightarrow \infty}\left(f^{\prime}\left(x_{i}\right)\right) & =\lim _{n \rightarrow \infty}\left(\frac{\widehat{x}-R}{\widehat{x}} \frac{H(\widehat{x}-R)}{g_{1} h_{i}(\widehat{x}-R)}\right) \\
& =\lim _{n \rightarrow \infty}\left(\frac{\widehat{x}-R}{\widehat{x}} \frac{1}{g_{1} k_{i}}\right) \\
\lim _{n \rightarrow \infty}\left(x_{i}\right) & =\lim _{n \rightarrow \infty} f^{\prime-1}\left(\frac{\widehat{x}-R}{\widehat{x}} \frac{1}{g_{1} k_{i}}\right)
\end{aligned}
$$

so that we have the desired result

$$
\lim _{n \rightarrow \infty} \frac{\widehat{x}}{n}=\lim _{n \rightarrow \infty} \frac{1}{n} \sum_{i=1}^{n} f^{\prime-1}\left(\frac{\widehat{x}-R}{\widehat{x}} \frac{1}{g_{1} k_{i}}\right)
$$

Note also that from equation (16) if $g_{1}=0$, it must be the case that $\lim _{n \rightarrow \infty}(\widehat{x}-R) / \widehat{x}=0$ so that $\lim _{n \rightarrow \infty} \widehat{x} / n=\rho$ 